\title{
Utilização da polpa de batata residual em snacks como perspectiva de redução do impacto ambiental
}

\author{
Thays de L. Dias ${ }^{1}$, Tatianne F. de Oliveira² ${ }^{2}$ Maria R. H. Campos ${ }^{3} \&$ Manoel S. Soares Júnior ${ }^{4}$ \\ ${ }^{1}$ EA/UFG. Goiânia, GO. E-mail: thaysld@hotmail.com \\ ${ }^{2}$ EA/UFG. Goiânia, GO. E-mail: ferreira.tatianne@yahoo.com.br \\ ${ }^{3}$ FANUT/UFG. Goiânia, GO. E-mail: raq7@brturbo.com.br \\ ${ }^{4}$ EA/UFG. Goiânia, GO. E-mail: mssoaresjr@hotmail.com (Autor correspondente)
}

Palavras-chave:

Solanum tuberosum L. resíduo industrial sustentabilidade agroindustrial

\begin{abstract}
R E S U M O
Neste trabalho se propôs caracterizar a polpa residual de lavagem de batatas desidratadas (PRLBD) e sua utilização em snacks fritos, visando à reutilização de resíduos gerados pelas agroindústrias. Amostras da água de lavagem de quatro cultivares de batata (Atlantic, Markies, Inovaiter e Asterix) foram coletadas e em seguida secadas. As características físicas e químicas das quatro PRLBD indicam que todas podem ser utilizadas como ingrediente na formulação de produtos alimentícios. Os snacks foram formulados com substituição parcial da farinha de trigo pela $\operatorname{PRLBD}(0,10,20$ e 50\%). Os resultados das análises microbiológicas realizadas com a PRLBD cultivar Atlantic, assim como os snacks, atendem aos requisitos da legislação brasileira não apresentando risco proeminente para a saúde humana (ausência de Salmonella; Coliformes a $45^{\circ} \mathrm{C}<100 \mathrm{UFC} \mathrm{g}^{-1}$ e Bacillus cereus $<3000 \mathrm{UFC} \mathrm{g}^{-1}$ ). As análises sensoriais mostraram que os snacks formulados foram os preferidos pelo consumidor, além de possuir um teor de lipídios $24 \%$ menor. Conclui-se que este novo processo de fabricação de snacks contribuirá para a sustentabilidade agroindustrial reduzindo os impactos ambientais gerados pelo processamento de batatas fritas, além de ser um produto mais saudável para o consumidor.
\end{abstract}

Key words:

Solanum tuberosum L. industrial residue agroindustrial sustainability

\section{Utilization of residual pulp of potato in snacks as prospect of reducing environmental impact}

\begin{abstract}
A B S T R A C T
The aim of this work was the characterization of a residual pulp of washing of dehydrated potato (PRLBD), as well as its use in snacks, with reuse of agroindustrial residue produced. Samples of the wash water from four cultivars of potato (Atlantic, Markies, Inovaiter and Asterix) were recovered and dried. The chemical and physical characteristics show that all PRLBD can be used as an ingredient in the formulation of food products. The snacks were formulated with partial substitution of wheat flour by PRLBD $(0,10,20 \mathrm{e} 50 \%)$. The results of microbiological analysis performed with the PRLBD cultivar Atlantic, such as the snacks were accepted by the Brazilian legislation and no significant risk to human health prominent (absence of Salmonella; Coliformes a $45^{\circ} \mathrm{C}<100 \mathrm{UFC} \mathrm{g}^{-1}$ and Bacillus cereus $<3000$ UFC. $\mathrm{g}^{-1}$ ). The acceptability analysis shows that the formulated snacks were most preferred by consumers, besides having a fat content $24 \%$ lower. In conclusion, this new process of manufacture of snacks can contribute to agroindustrial sustainability, decreasing the environment impact generated by processing chips, besides being a healthier product for the consumer.
\end{abstract}

\section{INTRODUÇÃo}

Nos últimos anos o setor de agroindústrias tem-se desenvolvido representando um importante papel no desenvolvimento da economia e gerando uma quantidade significativa de resíduos. Esses resíduos, sólidos ou líquidos, resultantes de atividades de processamento ou transformação de matéria-prima quando não tratados de forma correta, podem causar sérios problemas de poluição do solo, águas superficiais e subterrâneas (Roslev et al., 2007; Rigo et al., 2008; Oliveira et al., 2012).

Neste contexto a preocupação com o meio ambiente leva à viabilização de projetos que ressaltam a sustentabilidade nos sistemas de produção industrial. Nesta vertente se observa que a indústria de alimentos produz uma série de resíduos com alto valor de reutilização (Castro et al., 2011; Pinho e $t$ al., 2011; Souza et al., 2011; Barana et al., 2012). Inúmeros estudos utilizando resíduos agroindustriais têm sido realizados com o objetivo de minimizar o impacto ambiental das indústrias desenvolvendo alternativas de valorização de resíduos agroindustriais e de agregar valor aos produtos de mercado (Pelizer et al., 2007; Soares Júnior et al., 2011; Lima, 2011; Vilhalva et al., 2011; 2012; Sena et al., 2012; Castiglioni et al., 2013; Souza et al., 2013a,b; Sousa et al., 2013, Fiorda et al., 2013). 
Nas últimas duas décadas a indústria de processamento de batata vem ganhando espaço no cenário brasileiro abastecendo principalmente, o mercado de fastfood. No processamento industrial de tais batatas, a produção de resíduos ocorre nas operações de escolha, seleção e lavagem da matéria-prima (Fernandes et al., 2008). Entre esses resíduos se encontra a polpa residual da lavagem de batatas (PRLB) obtida após a etapa de fatiamento das batatas, durante o processo de lavagem; parte desta polpa é enviada diretamente para a Estação de Tratamento de Efluentes (ETE) da empresa e a outra parte passa por um préprocessamento (peneiramento e concentração) e posteriormente é acondicionada em big bags onde a polpa residual da lavagem de batata (PRLB) é decantada e a água filtrada; esta PRLB é um resíduo agroindustrial com potencial valor comercial, nutricional e tecnológico, que pode ser utilizado, assim como o amido de batata, em diversos seguimentos na indústria alimentícia (Santos, 2009).

Assim, este estudo teve, como objetivo, a determinação das propriedades físicas e químicas da PRLBD provenientes de diferentes cultivares de batata e seu aproveitamento na formulação de snacks fritos, pela substituição parcial da farinha de trigo e avaliar as propriedades físicas, químicas, microbiológicas e sensoriais desses novos produtos.

\section{Material e Métodos}

Foram coletadas amostras da polpa residual da lavagem de batata (PRLB) de quatro cultivares de batata (Atlantic, Markies, Inovaiter e Asterix) após as etapas de fatiamento e lavagem, na linha de processamento de batatas fritas da Empresa Cicopal Ltda., situada em Senador Canedo, Goiás, Brasil.

A água de lavagem das batatas fatiadas foi coletada em baldes de 20 L e deixada em repouso durante 20 min até sedimentação da PRLB; os experimentos de secagem da PRLB foram realizados em estufa de circulação e remoção de ar forçada a $60^{\circ} \mathrm{C}$ (Tecnal TE-394/3, Piracicaba-SP, Brasil), até atingir umidade ao redor de 14\%; após a secagem a PRLBDfoi acondicionada em sacos de polietileno.

Snacks sem PRLBD e com substituições de 10, 20 e 50\% de farinha de trigo (FT) por PRLBD foram formulados conforme descrito na Tabela 1. Utilizou-se delineamento inteiramente ao acaso com quatro tratamentos e cinco repetições. As etapas de fabricação foram: pesagem da matéria-prima, preparo da massa, fermentação a temperatura ambiente por $30 \mathrm{~min}$, cilindragem, corte, fermentação em estufa por $4 \mathrm{~min}$, fritura em óleo composto de soja e algodão $(80: 20 \mathrm{v} / \mathrm{v})$ por $2 \mathrm{~min} \mathrm{a}$ $180^{\circ} \mathrm{C}$, aromatização, embalagem e armazenamento. Todas as etapas do processamento dos snacks fritos foram realizadas na linha de produção da empresa Cicopal Ltda., situada em Senador Canedo, Goiás, Brasil.

$\mathrm{O} \mathrm{pH}$ das amostras de PRLBD e dos snacks fritos foi aferido utilizando-se potenciômetro (Genaka PG 1800); o teor de proteínas pelo método Kjeldahl para determinação do nitrogênio total, convertido em proteína bruta pelo fator 6,25; o teor de lipídios após a extração com éter de petróleo (obtido da Sigma Aldrich) em extrator Soxhlet; a umidade com a perda de peso da amostra, quando aquecida a $105^{\circ} \mathrm{C}$ em estufa com circulação de ar, até peso constante; o teor de cinzas por carbonização seguida de incineração completa em mufla a $550{ }^{\circ} \mathrm{C}$, todos seguindo os protocolos experimentais do Instituto Adolfo Lutz (IAL, 2008). Os carboidratos totais foram obtidos subtraindo-se de cem os valores obtidos de umidade, proteínas, lipídios e cinzas; as determinações dos parâmetros instrumentais de cor $\left(L^{*}, a^{*} e b^{*}\right)$ foram realizadas em colorímetro (Color Quest II, Hunter LabReston, Canadá), segundo método descrito por Paucar-Menacho et al. (2008); enfim, todas as análises foram realizadas em triplicata.

Contagem de Bacilluscereus, Coliformes totais e termotolerantes a $45^{\circ} \mathrm{C}$, tal como a pesquisa de presença de Salmonellas foi realizada de acordo com metodologia descrita pela American Public Health Association (APHA, 2001).

A análise sensorial dos snacks fritos foi realizada por 50 provadores treinados pela empresa Grasse Aromas e Ingredientes, Goiânia, Brasil, em cabines individuais. Foi realizado um teste de aceitação das amostras padrão e com $20 \%$ de substituição de FT por PRDLB, seguida de teste de preferência pareada, onde o provador escolhia a amostra preferida; no teste de aceitação foi utilizada escala hedônica de nove pontos: 9 - gostei muitíssimo, 5 - indiferente, 1 - desgostei muitíssimo. Protocolo número 117/11 aprovado pelo Comitê de Ética em Pesquisa da Universidade Federal de Goiás - UFG; no teste de preferência utilizou-se delineamento de blocos ao acaso em que cada provador foi considerado um bloco.

Os resultados obtidos foram submetidos a uma análise de variância (ANOVA) e as médias foram comparadas pelo teste Tukey ( $\mathrm{p} \leq 0,05)$, utilizando-se o aplicativo Statistica 7.0.

\section{Resultados E Discussão}

Pela Tabela 2 pode-se verificar que não houve diferença significativa entre as cultivares para lipídios, carboidratos e

Tabela 1. Formulação dos snacks fritos com diferentes níveis de substituição de farinha de trigo por polpa residual da lavagem da batata desidratada

\begin{tabular}{|c|c|c|c|c|}
\hline Ingrediente & \multicolumn{4}{|c|}{$\begin{array}{l}\text { Nível de substituição de farinha de trigo por polpa residual da lavagem } \\
\text { da batata desidratada (\%) }\end{array}$} \\
\hline Farinha de trigo - kg & 50 & 45 & 40 & 25 \\
\hline Fermento biológico - kg & 2 & 2 & 2 & 2 \\
\hline $\mathrm{Sal}(\mathrm{NaCl})-\mathrm{kg}$ & 1,5 & 1,5 & 1,5 & 1,5 \\
\hline Água - L & 36 & 36 & 36 & 36 \\
\hline
\end{tabular}


Tabela 2. Propriedades físicas e químicas da polpa residual da lavagem da batata em função da cultivar

\begin{tabular}{lrrrr}
\hline \multicolumn{1}{c}{ Propriedade } & \multicolumn{4}{c}{ Cultivar (cv.) } \\
\cline { 2 - 5 } Cinzas $\left(\mathrm{g} 100 \mathrm{~g}^{-1}\right)^{1}$ & \multicolumn{1}{c}{ cv. Asterix } & cv. Inovaiter & cv. Atlantic & \multicolumn{1}{c}{ cv. Markies } \\
Lipídios $\left(\mathrm{g} 100 \mathrm{~g}^{-1}\right)^{1}$ & $0,25 \mathrm{~b} \pm 0,01$ & $0,26 \mathrm{~b} \pm 0,010$ & $0,44 \mathrm{a} \pm 0,10$ & $0,28 \mathrm{~b} \pm 0,01$ \\
Proténa $\left(\mathrm{g} 100 \mathrm{~g}^{-1}\right)^{1}$ & $0,26 \mathrm{a} \pm 0,35$ & $0,36 \mathrm{a} \pm 0,290$ & $0,96 \mathrm{a} \pm 0,38$ & $0,99 \mathrm{a} \pm 0,20$ \\
Carboidratos $\left({\left.\mathrm{g} 100 \mathrm{~g}^{-1}\right)^{1}}^{0,35 \mathrm{ab} \pm 0,05}\right.$ & $0,37 \mathrm{ab} \pm 0,050$ & $0,31 \mathrm{~b} \pm 0,03$ & $0,47 \mathrm{a} \pm 0,05$ \\
Umidade $\left(\mathrm{g} 100 \mathrm{~g}^{-1}\right)^{1}$ & $81,10 \mathrm{a} \pm 0,37$ & $81,59 \mathrm{a} \pm 0,410$ & $81,29 \mathrm{a} \pm 0,13$ & $84,51 \mathrm{a} \pm 0,09$ \\
$\mathrm{pH}$ & $17,04 \mathrm{a} \pm 0,06$ & $17,42 \mathrm{a} \pm 0,130$ & $17,00 \mathrm{a} \pm 0,33$ & $13,75 \mathrm{a} \pm 0,12$ \\
Acidez titulável total (\%) & $6,15 \mathrm{c} \pm 0,17$ & $7,40 \mathrm{~b} \pm 0,120$ & $8,48 \mathrm{a} \pm 0,16$ & $6,32 \mathrm{c} \pm 0,07$ \\
$\mathrm{~L}^{*}$ & $0,73 \mathrm{~b} \pm 0,17$ & $0,29 \mathrm{c} \pm 0,037$ & $0,17 \mathrm{c} \pm 0,03$ & $1,63 \mathrm{a} \pm 0,19$ \\
$\mathrm{a}^{*}$ & $80,72 \mathrm{~d} \pm 0,34$ & $84,54 \mathrm{a} \pm 0,170$ & $82,19 \mathrm{c} \pm 0,24$ & $82,99 \mathrm{~b} \pm 0,21$ \\
$\mathrm{~b}^{*}$ & $0,18 \mathrm{a} \pm 0,01$ & $-0,20 \mathrm{~b} \pm 0,020$ & $-0,26 \mathrm{c} \pm 0,03$ & $-0,23 \mathrm{c} \pm 0,01$ \\
\hline
\end{tabular}

${ }^{1}$ Letras iguais na mesma linha significam que as médias não diferem a 0,05 de probabilidade; Obs.: Composição centesimal em base seca

$\mathrm{L}^{*}$ - luminosidade (preto ao braco) e coordenadas de cromaticidade; $\mathrm{a}^{*}$ - verde ao vermelho; $\mathrm{b}^{\star}$ - azul ao amarelo

umidade; no entanto, para cinzas, proteínas, $\mathrm{pH}$, acidez total, luminosidade (preto ao branco) e coordenadas de cromaticidade $a^{*}$ (verde ao vermelho) e $b^{*}$ (azul ao amarelo) foi constatada diferença entre as cultivares.

O teor de cinzas não diferiu entre as cultivares Atlantic e Markies nem entre as cultivares Asterix e Innovaiter mas variou $76,00 \%$ entre as cultivares com menor e maior nível, Asterix e Atlantic, respectivamente, estando de acordo com a legislação brasileira (Brasil, 1978), que preconiza um teor máximo $0,50 \%$ de cinzas para a fécula de batata. Em relação aos carboidratos houve variação de $4,20 \%$ entre as cultivares com menor e maior teor, Asterix e Markies, respectivamente. O nível de proteína da PRLBD diferiu entre as cultivares Markies e Atlantic sendo que as cultivares Asterix e Innovater não diferiram das demais. Ocorreu variação de 51,62\% no teor de proteína entre as cultivares com menor e maior nível, Atlantic e Markies, respectivamente; os resultados obtidos para lipídios revelam que a cultivar Asterix apresentou maior teor variando $250 \%$ em relação à cultivar Asterix, com menor nível. O teor de umidade variou $26 \%$ entre as cultivares com menor e maior nível, Markies e Innovaiter, respectivamente; os menores valores de $\mathrm{pH}$ foram observados nas PRLBD das cultivares Asterixe Markies, que não diferiram entre si embora tenham diferiram das demais, sendo maior na cultivar Atlantic; as amostras de PRLBD também se diferenciaram em relação à acidez total com exceção das cultivares Inovaiter e Atlantic que apresentaram valores menores e não se diferenciaram entre si $(p \geq 0,05)$.

Segundo a Tabela Brasileira de Composição de Alimentos (Taco, 2011), a farinha de trigo possui em média 13\% de umidade, $9,8 \%$ de proteína, $1,4 \%$ de lipídios, $75,1 \%$ de carboidratos e $0,8 \%$ de cinzas; já de acordo com Heineman et al. (1998) ela possui teor de cinzas de $0,74 \%, 77,22 \%$ de carboidratos e $13,44 \%$ de proteína. Portanto, a PRLBD possui valores semelhantes de umidade, maiores de carboidratos e menores de cinzas, lipídios e proteína, que a farinha de trigo. A Anvisa (Brasil,1978) prevê $14 \%$ de umidade e teor de cinzas de $0,50 \%$, para a fécula de batata e Fernandes et al. (2008) determinaram, para este mesmo produto, em média $9,72 \%$ de umidade, $5,56 \%$ de proteína e $2,22 \%$ de cinzas; assim, a PRLBD das diferentes cultivares é compatível com amidos e féculas tornando possível sua utilização como ingrediente na formulação dos snacks fritos considerando-se o ponto de vista químico.

Os parâmetros instrumentais de cor $\left(\mathrm{L}^{*}, \mathrm{a}^{*} \mathrm{e} \mathrm{b}^{*}\right)$ variaram significativamente entre as amostras; a luminosidade variou $4,73 \%$ entre as cultivares com menores e maiores níveis, Asterix e Innovaiter, confirmando a claridade das amostras ( 0 indica cor totalmente preta e 100 totalmente branca). Para a coordenada de cromaticidade $a^{\star}$ que representa a tonalidade que varia de verde a vermelho, e a coordenada $b^{\star}$, representadas pela variação de tonalidade do azul ao amarelo, os valores obtidos variaram pouco e foram próximos de zero, confirmando a tonalidade neutra da PRLBD passível de ser comparada com os resultados obtidos por Fernandes et al. (2008) em outros ingredientes, como a farinha de trigo e a farinha da casca da batata, amplamente utilizadas na formulação de produtos para consumo humano tais como biscoitos, bolos, pães.

Os resultados das análises microbiológicas da PRLBD da cultivar Atlantic e da água de lavagem de batatas em diferentes pontos de coleta, estão apresentados na Tabela 3. ausência de

Tabela 3. Resultado das análises microbiológicas da polpa residual de lavagem de batata desidratada (PRLBD) e da água de lavagem da cv. Atlantic e em snacks elaborados com diferentes níveis de substituição de farinha de trigo (FT) por polpa residual da lavagem da batata desidratada (PRLBD)

\begin{tabular}{|c|c|c|c|c|}
\hline \multirow{2}{*}{ Amostra } & \multicolumn{4}{|c|}{ Microrganismo } \\
\hline & Coliformes a $45^{\circ} \mathrm{C}$ & Coliformes Totais & Salmonella & Baciluscereus \\
\hline PRLBD Atantic & $<100$ UFC g $^{-1}$ & $2,6 \times 10^{2} \mathrm{UFC} \mathrm{g}^{-1}$ & Ausente em $25 \mathrm{~g}$ & $<3000 \mathrm{UFC} \mathrm{g}^{-1}$ \\
\hline Água da saída do tanque de lavagem & $<100$ UFC g $^{-1}$ & $5,2 \times 10^{3} \mathrm{UFC} \mathrm{g}^{-1}$ & Ausente em $25 \mathrm{~g}$ & $<3000$ UFC g $^{-1}$ \\
\hline Água da saída do filtro & $<100$ UFC g $^{-1}$ & $6,9 \times 10^{3} \mathrm{UFC} \mathrm{g}^{-1}$ & Ausente em $25 \mathrm{~g}$ & $<3000$ UFC g $^{-1}$ \\
\hline Snack frito padrão & $<50$ UFC g $^{-1}$ & - & Ausente em $25 \mathrm{~g}$ & - \\
\hline Snack frito com $10 \%$ PRLBD & $<50$ UFC g $^{-1}$ & - & Ausente em $25 \mathrm{~g}$ & - \\
\hline Snack frito com $20 \%$ PRLBD & $<50$ UFC g $^{-1}$ & - & Ausente em $25 \mathrm{~g}$ & - \\
\hline Snack frito com $50 \%$ PRLBD & $<50$ UFC g $^{-1}$ & - & Ausente em $25 \mathrm{~g}$ & - \\
\hline
\end{tabular}

UFC - Unidade formadora de colônias 


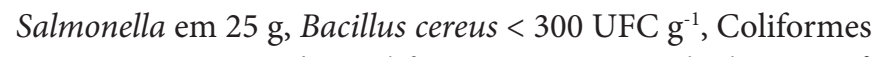
a $45^{\circ} \mathrm{C}<100 \mathrm{UFC} \mathrm{g}^{-1}$, e Coliformes totais variando de 2,6 $\times 10^{2}$ $\mathrm{UFC} \mathrm{g}^{-1}$ (PRLBD da cultivar Atlantic) a 9,6 $\times 10^{3} \mathrm{UFC} \mathrm{g}^{-1}$ (água de saída do filtro) foi verificada nas análises microbiológicas. Esses resultados estão de acordo com os padrões estabelecidos para fécula de batata (Brasil, 2001) sinalizando que a PRLBD é segura para utilização como ingrediente em substituição parcial de farinha de trigo na fabricação de snacks fritos.

Todas as propriedades físicas e químicas do snack padrão produzido pela empresa (controle) e dos formulados com diferentes níveis de substituição (10, 20 e 50\%) de farinha de trigo (FT) por PRLBD da cultivar Atlantic (escolhida por representar $90 \%$ da matéria-prima consumida na empresa) variaram significativamente (Tabela 4). Os teores de cinza, lipídios e proteína, foram maiores no snack controle enquanto o teor de carboidratos foi maior com $50 \%$ de substituição de FT por PRLBD. O teor de cinzas não variou significativamente entre os tratamentos controle, com 10 e $20 \%$ de substituição de FT por PRLBD, enquanto O de lipídios e de carboidratos dos tratamentos com 10 e $20 \%$ de substituição de FT por PRLBD não diferiu dos demais. $\mathrm{O}$ teor de proteína diminuiu com o nível de substituição de substituição de FT por PRLBD. Observou-se que o teor de cinzas variou $27,71 \%$, o de lipídios $42,31 \%$, o de proteína $51,49 \%$, o de carboidratos $25,53 \%$ e o de umidade $400 \%$, entre os snacks fritos com menor e maior nível de substituição.

Esses resultados mostraram que os snacks formulados com PRLBD absorvem menos gordura durante o processo de fritura provavelmente em função do menor tamanho dos grânulos (Yonemoto et al., 2007); neste trabalho o grânulo de amido da farinha de trigo é maior que os da PRLBD sendo, deste modo, mais saudável para o consumidor, embora os snacks também apresentem menor teor de proteína em virtude do teor elevado de proteína na farinha de trigo (Lobanco, 2007; Taco, 2011) quando comparado com o da PRLDB.

O snack padrão apresentou maior volume específico em comparação com os demais podendo ser observado visualmente conforme ilustra a Figura 1; as amostras com $10 \mathrm{e}$ 20\% de substituição de FT por PRLBD apresentaram formato semelhante, porém tamanho e diâmetro menores que a amostra padrão; a amostra com $50 \%$ de polpa de batata apresentou tamanho menor e maior diâmetro, em relação à amostra padrão. Esses resultados podem ser explicados devido ao teor de farinha

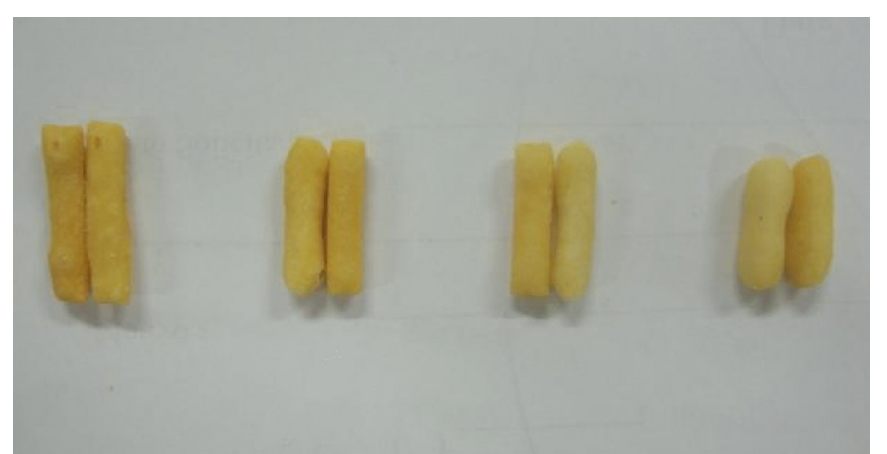

Figura. 1. Snacks fritos das diferentes formulações Padrão, 10, 20 e 50\% de polpa residual de lavagem de batata desidratada (PRLBD)

de trigo adicionado na formulação dos snacks. No snack com maior percentual de FT (padrão), provavelmente se formou uma rede de glúten mais forte, que conseguiu reter melhor os gases provenientes do metabolismo das leveduras produzidos na etapa de fermentação da massa (Scheuer et al., 2011).

Em relação à cor, o snack frito formulado com $10 \%$ de substituição de FT por PRLBD apresentou uma média menor de luminosidade (mais escuro), enquanto o com $20 \%$ foi o mais claro; todas as amostras se diferenciaram em relação a este parâmetro; as coordenadas de cromaticidade $\mathrm{a}^{*} \mathrm{e} \mathrm{b}^{*}$ obtiveram valores próximos de zero e mais elevados, respectivamente confirmando a tonalidade amarela dos snacks, conforme Figura 1. Os resultados das análises microbiológicas (Tabela 3) obtidos foram ausência de Salmonella em $25 \mathrm{~g}$ de amostra e Coliformes a $45{ }^{\circ} \mathrm{C}$ menor que 100 UFC por grama para todos os snacks fritos avaliados, atendendo aos padrões estabelecidos pela legislação (Brasil, 2001).

Os atributos aparência global, cor, odor e sabor dos snacks fritos avaliados no teste de aceitação não apresentaram diferenças significativas entre as médias atribuídas pelos provadores (Tabela 5). O tratamento com 20\% de substituição de FT por PRLBD apresentou médias mais elevadas em relação às da amostra padrão: $2,74,1,40,4,55$ e $5,55 \%$, respectivamente. Entretanto, em relação ao gosto residual (after taste), houve diferença significativa entre as médias dos dois snacks fritos e, segundo os resultados, a amostra padrão foi menos apreciada em relação ao sabor residual comparada com a amostra com 20\% de substituição de FT por PRLBD. Em referência ao teste

Tabela 4. Características físicas e químicas dos snacks padrão e dos formulados com diferentes níveis de substituição de farinha de trigo (FT) por polpa residual da lavagem da batata desidratada (PRLBD)

\begin{tabular}{|c|c|c|c|c|}
\hline \multirow{2}{*}{ Propriedade } & \multicolumn{4}{|c|}{ Nível de substituicão de farinha de triqo por polpa residual da lavaqem da batata desidratada (\%) } \\
\hline & 0 (controle) & 10 & 20 & 50 \\
\hline 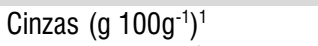 & $2,31 \mathrm{a} \pm 0,02$ & $2,35 \mathrm{a} \quad \pm 0,05$ & $2,33 \mathrm{a} \quad \pm 0,014$ & $1,84 b \pm 0,04$ \\
\hline Lipídios $\left(\mathrm{g}_{\left.100 \mathrm{~g}^{-1}\right)}\right.$ & $35,55 a \pm 3,71$ & $26,32 a b \pm 1,07$ & $27,11 a b \pm 4,050$ & $24,98 b \pm 5,26$ \\
\hline Proteínas $\left({\left.\mathrm{g} 100 \mathrm{~g}^{-1}\right)}^{-1}\right.$ & $8,12 a \pm 0,36$ & $7,14 b \pm 0,13$ & $7,23 b \pm 0,150$ & $5,36 \mathrm{c} \pm 0,87$ \\
\hline Carboidratos $\left({\left.\mathrm{g} 100 \mathrm{~g}^{-1}\right)}^{-1}\right.$ & $53,82 b \pm 4,08$ & $62,94 a b \pm 1,04$ & $62,28 a b \pm 3,840$ & $67,57 a \pm 5,31$ \\
\hline Umidade $\left(\mathrm{g} 100 \mathrm{~g}^{-1}\right)$ & $0,20 b \pm 0,03$ & $1,25 a \pm 0,11$ & $1,05 \mathrm{a} \pm 0,140$ & $0,25 b \pm 0,02$ \\
\hline Volume específico $\left(\mathrm{g} \mathrm{mL}^{-1}\right)$ & $0,37 a \pm 0,80$ & $0,28 c \pm 0,40$ & $0,22 d \pm 0,560$ & $0,33 b \pm 0,60$ \\
\hline$L^{*}$ & $45,32 c \pm 0,01$ & $43,91 d \pm 0,02$ & $49,21 \mathrm{a} \pm 0,010$ & $48,99 b \pm 0,01$ \\
\hline$a^{*}$ & $4,46 a \pm 0,03$ & $3,78 b \pm 0,01$ & $3,18 d \pm 0,020$ & $3,60 \mathrm{c} \pm 0,02$ \\
\hline$b^{*}$ & $11,44 c \pm 0,03$ & $10,20 d \pm 0,02$ & $12,35 b \pm 0,040$ & $13,83 a \pm 0,23$ \\
\hline
\end{tabular}

\footnotetext{
${ }^{1}$ Letras iguais na mesma linha significa que as médias não se diferem a 0,05 de significância ( $\left.p \leq 0,05\right)$; Obs: Composição centesimal em base seca
} $L^{*}$ - luminosidade (preto ao braco) e coordenadas de cromaticidade; $a^{*}$ - verde ao vermelho; $b^{\star}$ - azul ao amarelo 
Tabela 5. Teste de aceitação aplicado à formulação padrão e a formulação com $20 \%$ de polpa residual da lavagem da batata desidratada (PRLBD)

\begin{tabular}{lcc}
\hline \multirow{2}{*}{ Atributos } & \multicolumn{2}{c}{ Snacks fritos } \\
\cline { 2 - 3 } Aceitação global $^{1}$ & Padrão & 20\% PRLBD \\
Cor & $7,3 \mathrm{a}$ & $7,5 \mathrm{a}$ \\
Odor & $7,1 \mathrm{a}$ & $7,2 \mathrm{a}$ \\
Sabor & $6,6 \mathrm{a}$ & $6,9 \mathrm{a}$ \\
Gosto residual & $7,2 \mathrm{a}$ & $7,6 \mathrm{a}$ \\
Preferência (\%) & $6,8 \mathrm{~b}$ & $7,3 \mathrm{a}$ \\
\hline 'Letras iguais na mesma linha significa que as médias não se diferem a 0,05 de significância
\end{tabular}

de preferência, $70 \%$ dos provadores preferiram o snack frito formulado com $20 \%$ de substituição de FT por PRLBD sendo, portanto, um produto com forte potencial de comercialização.

\section{Conclusões}

1. O resíduo agroindustrial "polpa residual da lavagem da batata desidratada" não apresenta risco algum para o consumo humano podendo ser reaproveitado para ser utilizado como ingrediente na fabricação de snacks fritos, substituindo parcialmente a farinha de trigo.

2. Os snacks obtiveram características físicas, químicas, microbiológicas adequadas, além de apresentar um teor de lipídios inferior quando comparado com a amostra padrão comercial feita de farinha de trigo, sendo mais saudável para o consumidor.

3. Recomenda-se até $50 \%$ de substituição da farinha de trigo pela polpa residual da lavagem da batata desidratada.

\section{Agradecimentos}

À Fundação de Amparo à Pesquisa do Estado de Goiás (FAPEG) pelo apoio financeiro; ao Conselho Nacional de Desenvolvimento Científico e Tecnológico (CNPq) pela bolsa de produtividade em inovação tecnológica e extensão criativa, à Empresa Micos-Cicopal Ltda. pela parceria e ao Comitê de Ética da Universidade Federal de Goiás, protocolo $\mathrm{n}^{\circ}$ 117/2011, considerado em acordo com os princípios éticos vigentes.

\section{Literatura Citada}

APHA - American Public Health Association. Compendium of methods for the microbiological examination of foods. Washington: Public Health Association, v.4, 2001. 676p.

Barana, A. C.; Lima, R. C.; Botelhos, V. B.; Simões, D. R. Desenvolvimento de uma bebida láctea fermentada feita com soro ácido de queijo quark. Revista Verde de Agroecologia e Desenvolvimento Sustentável, v.7, p.13-21, 2012.

Brasil. Resolução CNNPA n.2, de 1978. Agência Nacional de Vigilância Sanitária. Normas técnicas gerais para alimentos. http://www.anvisa.gov.br. 8 Jan. 2013.
Brasil. Resolução RDC n.12, de janeiro de 2001. Agência Nacional de Vigilância Sanitária. Regulamento técnico sobre os padrões microbiológicos para alimentos. http//:www. anvisa.gov.br.8 Jan. 2013.

Castiglioni, G. L.; Soares Júnior, M. S.; Caliari, M.; Silva, F. A. Modelagem matemática do processo de secagem da massa fibrosa de mandioca. Revista Brasileira de Engenharia Agrícola e Ambiental, v.17, p.987-994, 2013.

Castro, I. P. M.; Alvim, T. C.; Santana, W. R.; Carvalho, D. P.; Silveira, M. A. Efeito da adição de soro de queijo no processo de obtenção de etanol a partir de batata-doce. Ciência Agrotécnica, v.35, p.980-986, 2011.

Fernandes, A. F.; Pereira, J.; Germani, R.; Oiano Neto, J. Effect of the parcial replacement of wheat flour for potato skin flour (Solano Tuberosum L.). Ciência e Tecnologia de Alimentos, v.28, p.56-65, 2008.

Fiorda, F. A.; Soares Júnior, M. S.; Silva, F. A.; Grosmann, M. V. E.; Souto, L. R. F. Microestructure, texture and colour of gluten-free pasta made with amaranth flour, cassava starch and cassava bagasse. Lebensmittel-Wissenschaft + Technologie/Food Science + Technology, v.54, p.132-138, 2013.

Heinemann, R. B.; Costa, N. M. B.; Cruz, R.; Pirozi, M. R. Nutritional value of wheat flour mixed with cassava leaf protein concentrate. Revista Nutrição, v.11, p.51-57, 1998.

IAL - Instituto Adolf Lutz, Métodos Físico-Químicos para Análise de Alimentos. 4.ed. São Paulo: IAL, 2008. 994p.

Lima, C. C. Disponibilidade de fósforo para a cana-de-açúcar em solo tratado com compostos orgânicos ricos em silício. Revista Brasileira de Engenharia Agrícola e Ambiental, v.15, p.1222-1227, 2011.

Lobanco, C. M. Rotulagem nutricional de alimentos salgados e doces consumidos por crianças e adolescentes, São Paulo: UFSP, 2007. 107p. Dissertação Mestrado

Oliveira, F. T.; Cagnon, B.; Fauduet, H.; Licheron, M.; Chedeville, O. Removal of diethyl phthalate from aqueous media by adsorption on different activated carbons: Kinetic and isotherm studies. Separation Science and Technology, v.47, p.1139-1148, 2012.

Paucar-Menacho, L. M.; Barreto, P. A. A.; Mazal, G.; Fakhouri, F. M.; Steel, C. J.; Collares-Queiroz, F. P. Desenvolvimento de massa alimentícia fresca functional com a adição de isolado protéico de soja e polidextrose utilizando páprica como corante. Ciência e Tecnologia de Alimentos, v.28, p.767-778, 2008.

Pelizer, L. H.; Pontineri, M. H.; Moraes, I. Utilização de resíduos agro-industriais em processos biotecnológicos como perspectiva de redução do impacto ambiental. Journal of Technology Management \& Innovation, v.2, p.118-127, 2007.

Pinho, L. X.; Afonso, M. R. A.; Carioca, J. O. B.; Costa, J. M. C.; Rybka, A. C. P. Desidratação e aproveitamento de resíduo de pedúnculo de caju como adição de fibra na elaboração de hambúrguer. Alimentos e Nutrição, v.22, p.571-576, 2011.

R. Bras. Eng. Agríc. Ambiental, v.18, n.2, p.225-230, 2014. 
Rigo, E.; Rigoni, R. E.; Lodea P.; Oliveira, D.; Freire, D. M. G.; Di Luccio, M. Application of different lipases as pretreatment in anaerobic treatment of wastewater. Environmental Science Engineering, v.25, p.1243-1249, 2008.

Roslev, P.; Vorkamp, K.; Aarup, J.; Frederiksen, K.; Nielsen, P. $\mathrm{H}$. Degradation of phthalate esters in an activated sludge wastewater treatment plant. Water Research, v.41, p.969976, 2007.

Santos, A. P. Farinha de batata (Solanum tuberosum L.): Obtenção, caracterização físico-química, funcional, elaboração e caracterização de sopas desidratadas. Itapetinga: Universidade Estadual do Sudoeste da Bahia, 2009. 105p. Dissertação Mestrado

Scheuer, P. M.; Francisco, A.; Miranda, M.Z.; Limberger, V.M. Trigo: Características e utilização na panificação. Revista Brasileira de Produtos Agroindustriais, v.13, p. 211-222, 2011.

Sena, R. F.; Albrecht, W.; Althoff, C. A.; Moreira, R. F. P. M.; José, $\mathrm{H}$. J. Characterisation of agroindustrial solid residues as biofuels and potential application in thermochemical processes. Waste Management, v.32, p.1952-161, 2012.

Soares Júnior, M. S.; Santos, T. P. B.; Pereira, G. F.; Minafra, C. S.; Caliari, M.; Silva, F. A. Development of extruded snacks from rice and bean fragments. Semina: Ciências Agrárias, v.32, p.189-198, 2011.

Sousa, G. M.; Soares Júnior, M. S.; Yamashita, F. Active biodegradable films produced with blends of rice flour and poly (butylene adipate co-terephthalate): Effect of potassium sorbate on film characteristics. Materials Science \& Engineering. C, Biomimetic Materials, Sensors and Systems, v.33, p.3153-3159, 2013.
Souza, A. R. M.; Silva, Y. P. A.; Costa, N. V.; Almeida, T. L.; Arthur, V.; Lage, M. L.; Asquieri, E. R.; Damiani, C. Irradiação em barra de cereais incorporadas com casca de abacaxi. Pesquisa Agropecuária Tropical, v.41, p.610-614, 2011.

Souza, T. A. C.; Soares Júnior, M. S.; Campos, M. R. H.; Souza, T. S. C.; Bandeira, L. C. The effect of chemical treatments on the $\mathrm{pH}$ and microbial flora of cassava residues during storage. Ciência e Tecnologia de Alimentos, v.33, p.500507, 2013a.

Souza, T. A. C.; Soares Júnior, M. S.; Souza, T. S. C.; Campos, M. R. H. Bolos sem glúten a base de arroz quebrado e casca de mandioca. Semina. Ciências Agrárias, v.34, p.717-728, 2013b.

TACO - Tabela Brasileira de Composição de Alimentos. UNICAMP, 4.ed, 2011.p.25-32. http://www.unicamp.br.br/ nepa/taco. 8 Jan. 2013.

Vilhalva, D. A. A.; Soares Júnior, M. S.; Caliari, M.; Silva, F. A. Secagem convencional de casca de mandioca proveniente de resíduos de indústria de amido. Pesquisa Agropecuária Tropical, v.42, p.331-339, 2012.

Vilhalva, D. A. A.; Soares Júnior, M. S.; Moura, C. M. A.; Caliari, M.; Souza, T. A. C.; Silva, F. A. Aproveitamento da farinha de casca de mandioca na elaboração de pão de forma. Revista do Instituto Adolfo Lutz, v.70, p.514-521, 2011.

Yonemoto, P. G.; Calori-Domingues, M. A.; Franco, C. M. L. Effect of granule size on the structural and physicochemical characteristics of wheat starch. Food Science and Technology, v.27, p.761-771, 2007. 